and the timing of treatments, an inescapable conclusion is that we still have an incomplete understanding of the critical host-pathogen interactions that cause the local and systemic manifestations of sepsis in humans.

The MIF and sepsis lines of investigation intersected when Donnelly and associates showed that MIF was detectable in the serum and the lung fluids of patients with the acute respiratory distress syndrome (ARDS) ${ }^{10}$. ARDS results from severe and diffuse lung injury in which the epithelial and endothelial barriers of the alveolar walls are severely injured, the airspaces fill with exudative fluid and efficient gas exchange fails ${ }^{11}$. Risk factors include sepsis, severe trauma and aspiration of gastric contents, and the mortality ranges from about 35 to $50 \%$ in different centers $^{12}$. MIF was detectable in alveolar macrophages of patients with ARDS, and MIF inhibited the suppressive effects of dexamethasone on IL-8 production by lung macrophages, consistent with earlier experimental results. This was the first clinical evidence to suggest that the effects of MIF identified in basic studies might be relevant for humans with lifethreatening illness, moving MIF from the bench toward the bedside.

The results of the study by Calandra $e t$ al. show that MIF has an essential function in mice with bacterial peritonitis, induced either by ligation and puncture of the cecum, or by the direct intraperitoneal injection of E. coli. Cecal ligation and puncture (CLP) produces a serious intraabdominal infection with many features of human septic shock. Unlike sustained intravascular infusion of bacteria or their products, which seldom occurs in humans, CLP produces a relevant model of a complex tissue infection that occurs in humans.

Calandra et al. report that neutralization of MIF prevented death in the CLP model, even when treatment with antibody against MIF was begun as long as 8 hours after the onset of peritonitis. This is the only example of a substantial posttreatment effect of an anti-cytokine therapy in a tissue model of infection. The authors have also made the salient observation that the antibody against MIF protected TNF- $\alpha$ knockout mice from lethal peritonitis, indicating that the effects of MIF in CLP are independent of TNF- $\alpha$, and suggest that MIF has a major effect in determining the outcome of peritonitis and sepsis. Additionally, anti-MIF treatment of animals with $E$. coli peritonitis re- sulted in lower concentrations of circulating bacteria and TNF- $\alpha$ at $12 \mathrm{~h}$ after CLP, consistent with an improvement in host defenses. This unexpected effect seems paradoxical in part, as neutralizing MIF might be expected to accentuate the activity of endogenous glucocorticoids, with potentially adverse effects on antibacterial defenses.

It is premature to conclude that MIF is the essential mediator of sepsis in humans. More information is needed about the cellular mechanisms by which MIF acts to understand where MIF fits in the complex network of events that occurs in sepsis. Nevertheless, an unexpected line of investigation that began with a very simple question about what inhibits the effects of cortisol has placed MIF at the center of a new paradigm about the essential events in the pathogenesis of sepsis. Human studies are underway that should provide a clinical perspective about the importance of MIF in sepsis and other human diseases.

1. Bloom, B.R. \& Bennett, B. Mechanism of a reaction in vitro associated with delayed-type hypersensitivity. Science 153, 80-82 (1966).

2. David, J.R. Delayed hypersensitify in vitro: its mediation by cell-free substances formed by lymphoid cell-antigen interaction. Proc. Natl. Acad. Sci. USA 56, 72-77 (1966)

3. Bernhagen, J. et al. MIF is a pituitary-derived cy- tokine that potentiates lethal endotoxemia. Nature 365, 756-759 (1993)

4. Calandra, T. et al. MIF as a glucocorticoid-induced modulator of cytokine production. Nature 377, 68-71 (1995).

5. Weiser, W.Y et al Molecular cloning of a CDNA encoding a human macrophage migration inhibitory factor. Proc. Natl. Acad. Sci. USA 86, 7522-7526 (1989).

6. Calandra, T. et al. Protection from septic shock by neutralization of macrophage migration inhibitory factor (MIF). Nature Med. 6, 164-170 (2000).

7. Fowler, A.A., III et al. Adult respiratory distress syndrome: risk with common predispositions. Ann. Intern. Med. 98, 593-597 (1983).

8. Montgomery, A.B., Stager, M.A., Carrico, C.J. \& Hudson, L.D. Causes of mortality in patients with the adult respiratory distress syndrome. Am. Rev. Respir. Dis. 132, 485-489 (1985).

9. Abraham, E. Why immunomodulatory therapies have not worked in sepsis. Intensive Care Med. 25 556-566 (1999).

10. Donnelly, S.C. et al. Regulatory role for macrophage migration inhibitory factor in the acute respiratory distress syndrome. Nature Med. 3, 320-323 (1997).

11. Bernard G.R et al. The American-European Consensus Conference on ARDS: Definitions, mechanisms, relevant outcomes and clinical trial coordination. Am. J. Respir. Crit. Care Med. 149, 818-824 (1994).

12. Hudson, L.D., Milberg, J.A., Anardi, D. \& Maunder, R.J. Clinical risks for development of the acute respiratory distress syndrome. Am. J. Respir. Crit. Care Med. 151, 293-301 (1995).

\section{The Seattle VA Medical Center}

Department of Medicine

University of Washington

Seattle, Washington 98108, USA

Email: trmartin@u.washington.edu

\title{
Oral examination
}

Oral squamous cell carcinomas (OSCCs) are characterized by complex, often near-triploid karyotypes that involve large numbers of chromosomal deletions, translocations and structural abnormalities. The laboratories of William Saunders (University of Pittsburg) and Susanne Gollin (University-Hospital Charite, Berlin) investigated the cellular mechanisms of chromosomal loss and rearrangement during mitosis using

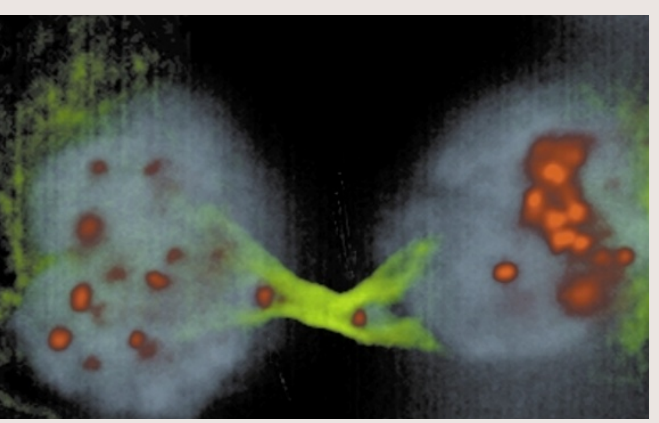
fluorescence microscopy. In a paper published in the 4 January issue of PNAS, they report that during metaphase, OSCC cells often develop multipolar spindles, leading to errors in chromosome segregation, such as the formation of 'lagging' chromosomes that are pulled in multiple directions between dividing cells. The image shows that during telophase, these chromosomes often become trapped in an 'anaphase' bridge that contains tubulin (yellow) and a few remaining centromeres (red), which persist between dividing nuclei (blue). During this stage of normal cell division, the chromosomes should already be fully segregated and the nuclei should be re-forming. In OSCC cells, however, the lagging chromosomes are excluded from the re-forming nuclei and incorporated into micronuclei. Micronuclei are often seen in oral cancer cells, and are an important biomarker for this disease. The authors suggest that cytoskeletal defects might be responsible for the abnormal chromosome segregation and genetic instability of oral cancer cells.

Kristine Novak 